\title{
Isoprenoid biosynthesis in dandelion latex is enhanced by the overexpression of three key enzymes involved in the mevalonate pathway
}

Katharina M. Pütter ${ }^{1}$, Nicole van Deenen ${ }^{1}$, Kristina Unland ${ }^{2}$, Dirk Prüfer ${ }^{1,2}$ and Christian Schulze Gronover ${ }^{2^{*}}$ (D)

\begin{abstract}
Background: Latex from the dandelion species Taraxacum brevicorniculatum contains many high-value isoprenoid end products, e.g. triterpenes and polyisoprenes such as natural rubber. The isopentenyl pyrophosphate units required as precursors for these isoprenoids are provided by the mevalonate (MVA) pathway. The key enzyme in this pathway is 3hydroxy-methyl-glutaryl-CoA reductase (HMGR) and its activity has been thoroughly characterized in many plant species including dandelion. However, two enzymes acting upstream of HMGR have not been characterized in dandelion latex: ATP citrate lyase (ACL), which provides the acetyl-CoA utilized in the MVA pathway, and acetoacetylCoA thiolase (AACT), which catalyzes the first step in the pathway to produce acetoacetyl-CoA. Here we isolated $A C L$ and AACT genes from T. brevicorniculatum latex and characterized their expression profiles. We also overexpressed the well-characterized HMGR, ACL and AACT genes from Arabidopsis thaliana in T. brevicorniculatum to determine their impact on isoprenoid end products in the latex.

Results: The spatial and temporal expression profiles of T. brevicorniculatum ACL and AACT revealed their pivotal role in the synthesis of precursors necessary for isoprenoid biosynthesis in latex. The overexpression of A. thaliana ACL and AACT and HMGR in T. brevicorniculatum latex resulted in the accumulation of all three enzymes, increased the corresponding enzymatic activities and ultimately increased sterol levels by $\sim 5$-fold and pentacyclic triterpene and cis-1,4-isoprene levels by $\sim 2$-fold. Remarkably high levels of the triterpene precursor squalene were also detected in the triple-transgenic lines (up to $32 \mathrm{mg} / \mathrm{g}$ root dry weight) leading to the formation of numerous lipid droplets which were observed in root crosssections.

Conclusions: We could show the effective expression of up to three transgenes in T. brevicorniculatum latex which led to increased enzymatic activity and resulted in high level squalene accumulation in the dandelion roots up to an industrially relevant amount. Our data provide insight into the regulation of the MVA pathway in dandelion latex and can be used as a basis for metabolic engineering to enhance the production of isoprenoid end products in this specialized tissue.
\end{abstract}

Keywords: ATP citrate lyase, Acetoacetyl-CoA thiolase, Mevalonate pathway, Taraxacum, Natural rubber, Latex, Triterpenes, Sterols

\footnotetext{
* Correspondence: christian.schulze.gronover@ime.fraunhofer.de

${ }^{2}$ Fraunhofer Institute for Molecular Biology and Applied Ecology (IME),

Schlossplatz 8, 48143 Muenster, Germany

Full list of author information is available at the end of the article
} 


\section{Background}

The dandelion species Taraxacum brevicorniculatum belongs to more than 12,500 plant species that produce latex, a milky sap, in specialized parenchyma cells called laticifers [1]. Among other secondary metabolites and proteins, laticifers contain a large variety of industrially valuable isoprenoid end products, including triterpenes such as $\alpha$-amyrin, $\beta$-amyrin, lupeol and taraxasterol, and polyisoprenes such as natural rubber [2]. The components of the rubber transferase complex have been investigated in rubber-producing plants like the rubber tree (Hevea brasiliensis), lettuce (Lactuca sativa) and $T$. brevicorniculatum [3-5]. Labeling experiments in $H$. brasiliensis showed that the cytosolic mevalonate (MVA) pathway provides the precursor isopentenyl pyrophosphate (IPP) for rubber biosynthesis in the laticifers [6, 7]. We therefore characterized the key enzymes of the MVA pathway and upstream reactions in T. brevicorniculatum latex [see Fig. 1].

In several previous studies, 3-hydroxy-methyl-glutaryl-CoA reductase (HMGR) has been identified as the rate-limiting enzyme in the MVA pathway [8-12]. Plant HMGRs are characterized by four domains: a variable cytosolic $\mathrm{N}$-terminus, two endoplasmic reticulum transmembrane domains, a variable region between the transmembrane domains, and a cytosolic $\mathrm{C}$-terminus containing the highly-conserved catalytic domain [13, 14]. Plant HMGRs are regulated at the post-transcriptional and post-translational levels, e.g. by phosphorylation and inhibition by their own N-terminus [15-18]. T. brevicorniculatum produces three HMGRs encoded by the genes TbHMGR1, TbHMGR2 and TbHMGR3, with TbHMGR1 predominantly expressed in the latex [19]. MVA pathway engineering in plants is challenging $[12,20]$. The overexpression of endogenous key enzymes often fails to increase the yield of end products due to negative feedback or ratelimiting downstream steps, and the regulatory mechanisms therefore need to be characterized in more detail $[12,21]$.

Enzymes acting upstream of HMGR in the latex might act as additional rate-limiting steps for isoprenoid biosynthesis, including ATP citrate lyase (ACL) and acetoacetyl-CoA thiolase (AACT) [see Fig. 1]. Acetyl$\mathrm{CoA}$ is required in many anabolic and catabolic pathways in plants and must be synthesized in every cell compartment [22]. ACL cleaves citrate into oxaloacetate and acetyl-CoA in the cytosol. It belongs to the thiokinase superfamily, which also includes succinyl-CoA synthetase (SCS) and citrate synthase (CS) among others [23]. SCS is localized in mitochondria and catalyzes the reversible conversion of succinyl-CoA to succinate and CoA. Therefore, ATP-binding and CoA-binding domains are conserved between SCS and ACL. Arabidopsis thaliana $\mathrm{ACL}$ is a $500 \mathrm{kDa}$ heteromeric protein comprised of

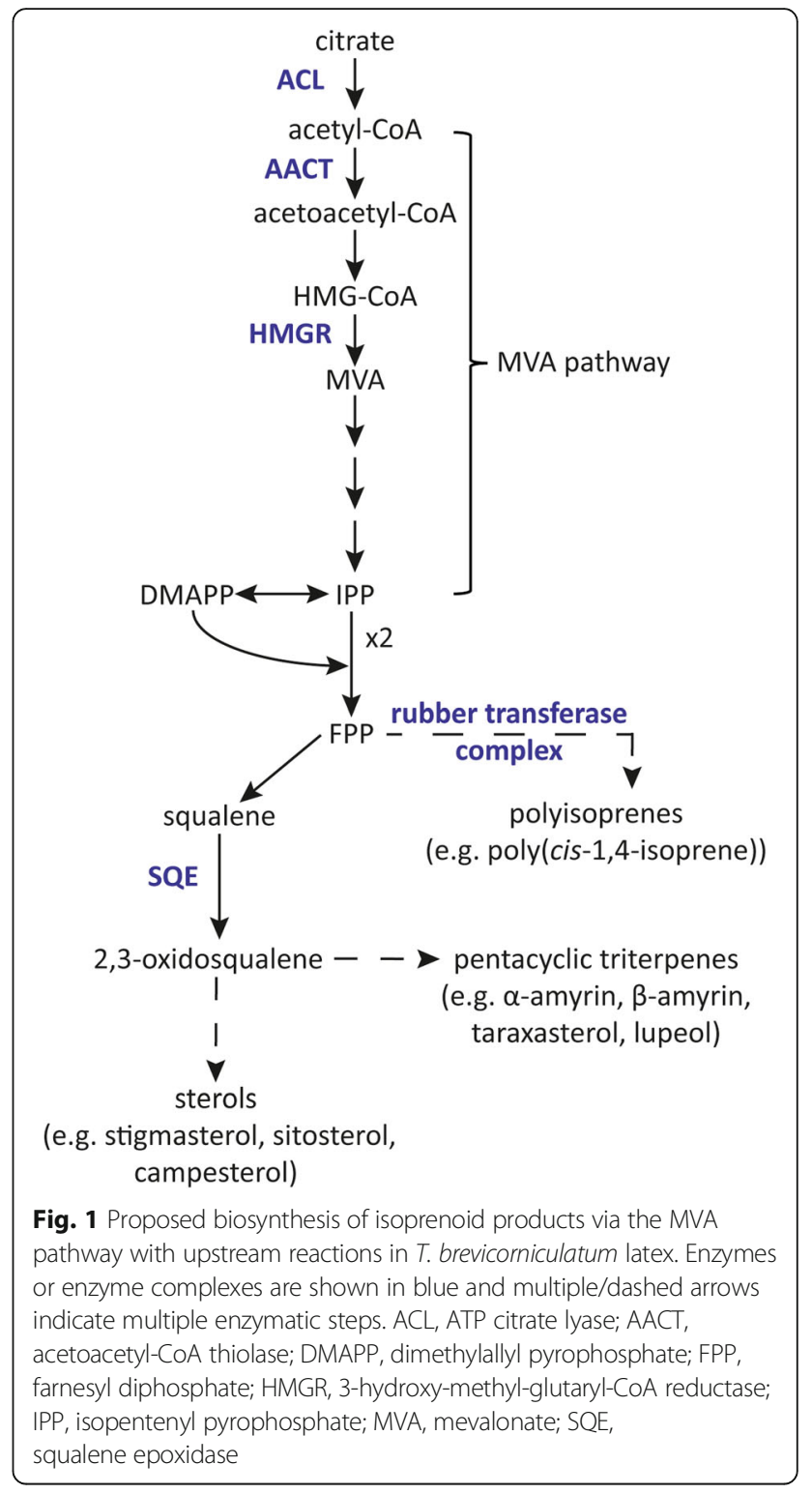

ACLA and ACLB subunits. AtACLA is very similar to the $\beta$-subunit of AtSCS, whereas AtACLB is more closely related to the $\alpha$-subunit of AtSCS and to AtCS [24]. Transgenic A. thaliana plants expressing AtACLA1 antisense RNA have a severe dwarf phenotype which underlines the importance of this enzyme for anabolic processes [25]. Furthermore, the overexpression of an $A$. thaliana AtACL(AB) fusion protein in dandelion latex increased ACL activity and led to the accumulation of various isoprenoid end products, highlighting its ability to increase flux through the MVA pathway [26]. The effective shuttling of acetyl-CoA into the MVA pathway requires a Claisen-type condensation reaction between two acetyl-CoA molecules to form acetoacetyl-CoA, which is catalyzed by AACT. In plants, catabolic 3ketoacyl-CoA thiolases (type I thiolases; EC 2.3.1.16) and 
anabolic acetoacetyl-CoA thiolases (type II thiolases; EC 2.3.1.9) belong to the thiolase superfamily [27]. The MVA pathway requires type II thiolases, and the corresponding enzymes have been characterized in A. thaliana, $H$. brasiliensis and Medicago sativa. In A. thaliana, the AtAACT1 and AtAACT2 genes have different spatiotemporal expression profiles and knockout phenotypes: Ataact1 T-DNA knockout lines show normal growth and development whereas Ataact2 T-DNA knockout lines are embryonic lethal [28]. Furthermore, AtAACT2 RNAi lines are sterile and are characterized by a reduced cell size and cell number, indicating that AtAACT2 is essential among others for sterol biosynthesis via the MVA pathway, which is pivotal for membrane biosynthesis. In $H$. brasiliensis, $H b A A C T 1$ is predominantly expressed in the latex whereas $H b A A C T 2$ and $H b A A C T 3$ are mainly expressed in mature leaves [7]. In $M$. sativa, the key role of MsAACT1 in isoprenoid biosynthesis was shown by overexpression experiments, which resulted in higher thiolase activity and the accumulation of squalene [29].

The dandelion $A C L$ and $A A C T$ genes have not been characterized in detail but may play a key role in isoprenoid biosynthesis as in other plants. We therefore identified $A C L$ and $A A C T$ genes predominantly expressed in T. brevicorniculatum latex and characterized their spatiotemporal expression profiles. We also overexpressed the wellcharacterized A. thaliana HMGR, ACL and AACT genes in $T$. brevicorniculatum to increase flux through the MVA pathway predominantly in the latex. This allowed us to determine the impact of the three enzymes on the accumulation of isoprenoid end products such as triterpenes and polyisoprenes.

\section{Methods}

\section{Plant material and cultivation conditions}

T. brevicorniculatum seeds were obtained from the Botanical Garden Marburg (Germany) and were cultivated at $18{ }^{\circ} \mathrm{C}$ and 20 klux with a 16 -h photoperiod in controlled growth chambers or in the greenhouse. Plants were cultivated in a pre-fertilized 1:1 mixture of standard soil (ED73 Einheitserde, Fröndenberg, Germany) and garden mold (Botanical Garden Münster, Germany). They were fed every 4 weeks with a commercial fertilizer according to the manufacturer's recommendations (Hakaphos Plus, Compo GmbH, Münster, Germany). Arabidopsis thaliana ecotype Columbia (Col-0) seeds were acquired from the Nottingham Arabidopsis Stock Centre (University of Nottingham, Loughborough, United Kingdom, http://arabidopsis.info) and cultivated as stated above. Seeds of Nicotiana benthamiana laboratory isolate (LAB) [30] were obtained from the Sainsbury Laboratory (John Innes Centre, Norwich, United Kingdom) and cultivated as stated above.

\section{RNA extraction and CDNA synthesis}

Total RNA was extracted from T. brevicorniculatum root latex using the RNeasy Lipid Tissue Kit (Qiagen, Hilden, Germany), and from T. brevicorniculatum root, peduncle, leaf and flower tissues using the innuPREP RNA Mini Kit (Analytik Jena, Jena, Germany), each according to the manufacturer's instructions. Full-length cDNA was synthesized from 500 ng total RNA using PrimeScript RT Master Mix (TaKaRa, Clontech, Saint-Germain-en-Laye, France) according to the manufacturer's instructions. Fulllength CDNA sequences were obtained by standard RACE procedures. Oligonucleotide sequences are shown in Additional file 1 [see Additional file 1].

\section{Cloning and transformation procedures}

Expression vector pLab12.10-pREF-AtACL(AB) which contained the promoter of the rubber elongation factor (REF) was prepared as previously described [26]. The AtHMGR1(S408A) catalytic domain with a mutated phosphorylation site at position 408 was amplified from $A$. thaliana leaf cDNA using primers Athmgrc1ATG-Pcilfwd and Athmgrc1(S408A)-XbaI-rev, digested with PciI/ $\mathrm{XbaI}$ and inserted at the NcoI/XbaI sites of pLab12.1pREF [5] resulting in final vector pLab12.1pREF-Athmgr c1(S408A). The same fragment was inserted into the pCambia1305.1 vector as previously described [19]. Expression of heterologous AtHMGR variants pCambia 1305.1-Athmgrc1 and pCambia1305.1-Athmgrc1(S408A) in Nicotiana benthamiana was carried out according to van Deenen et al. [19]. The Agrobacterium tumefaciens strain GV3101pMP90RK used for infiltration was obtained from DNA Cloning Service e.K. (Hamburg, Germany). The AtAACT2 sequence was amplified from the same source using primers AtAACT2-Sall-fwd and AtAACT2-XbaI-rev, digested with SalI/XbaI and inserted at the $\mathrm{Xhol} / \mathrm{Xbal}$ sites of the same plasmid resulting in the final vector pLab12.1pREF-AtAACT2. An expression vector containing both sequences was prepared by removing the pREF-Athmgrc1(S408A) cassette from pLab12.1pREF-Athmgrc1(S408A) using HindIII/BamHI and transferring it to the PmeI site in pLab12.1pREF-AtA ACT2 to yield the final vector pLab12.1-pREF-AtAACT2pREF-Athmgrc1(S408A). The transformation of T. brevicorniculatum by Agrobacterium tumefaciens strain EHA 105 provided by Beth Hood, Prodigene Inc., College Station, TX, USA [31] was carried out as previously described [32]. The constructs pLab12.1pREF-Athmgrc1(S408A) and pLab12.1pREF-AtAACT2 were introduced into wildtype $T$. brevicorniculatum plants, whereas pLab12.1pREFAtAACT2-pREF-Athmgrc1(S408A) was introduced into the transgenic $T$. brevicorniculatum line TbAB1 expressing the pREF-AtACL(AB) construct [26]. Oligonucleotide sequences are shown in Additional file 1. 


\section{Quantitative RT-PCR (qRT-PCR)}

Quantitative RT-PCR analysis was carried out as previously described [26] with slight modifications. Latex was harvested from 8-week-old roots of wild-type and transgenic T. brevicorniculatum plants for RNA isolation, cDNA synthesis and qRT-PCR using the KAPA SYBR FAST qPCR Master Mix (Peqlab, Erlangen, Germany) in a Bio-Rad iCycler real-time PCR system. Three different $T$. brevicorniculatum reference genes were included in the qRT-PCR analysis: The glyceraldehyde-3-phosphate dehydrogenase (TbGAPDH) and the elongation factor-1 alpha $(T b E F 1 \alpha)$ as already described [26, 33], as well as the ribosomal protein L27 (TbRP) based on an expressed sequence tag (EST) (GenBank GO664824) of the T. koksaghyz root cDNA library. The relative expression levels of each target gene were calculated using Bio-Rad CFX Manager 3.1 software (Bio-Rad Laboratories Inc., Hercules, USA). All oligonucleotide sequences for the reference, endogenous and transgene expression analysis are shown in Additional file 1. Primer efficiencies, amplification factors and usage of formamide for enhancing primer specificity are shown in Additional file 2.

\section{SDS-PAGE and western blot analysis}

Taraxacum brevicorniculatum protein was extracted and analyzed as previously described [26] with slight modifications. The rubber extraction buffer contained $100 \mathrm{mM}$ Tris (pH 7.5), $350 \mathrm{mM}$ sorbitol, $10 \mathrm{mM} \mathrm{NaCl}, 5 \mathrm{mM}$ $\mathrm{MgCl}_{2}$, and $5 \mathrm{mM}$ dithiothreitol (DTT). A rabbit antibody against $A$. thaliana ACLB was kindly provided by Yves Poirier (Department of Plant Molecular Biology, Université de Lausanne, Switzerland) and was diluted 1:2000 before use. A rabbit antibody against the A. thaliana HMGR1 catalytic domain was kindly provided by Hubert Schaller (Institut de Biologie Moléculaire des Plantes, Université de Strasbourg, France) and diluted 1:7500 before use. Both were detected with a secondary alkaline phosphatase (AP)-conjugated goat anti-rabbit IgG antibody diluted 1:10,000 before use. A mouse antibody against $A$. thaliana AACT2 was kindly provided by Basil Nikolau (Roy J. Carver Department of Biochemistry, Biophysics and Molecular Biology, Iowa State University, USA) and was diluted 1:1000 before use as previously described [28]. This was detected with a secondary APconjugated goat anti-mouse IgG antibody diluted 1:10,000 before use.

\section{Analysis of enzyme activity}

ACL activity in dandelion latex from 8-week-old roots of $T$. brevicorniculatum plants was determined as previously described [26]. AACT activity was determined by measuring the reduction of 5,5'-dithiobis-(2-nitrobenzoic acid) (DTNB) by free CoA as previously described [34] with slight modifications. We incubated $5 \mu \mathrm{g}$ of crude protein extracted from the latex of 8-week-old roots of $T$. brevicorniculatum plants in $95 \mu \mathrm{l}$ reaction buffer $(50 \mathrm{mM}$ Tris/ $\mathrm{HCl}, 0.5 \mathrm{M}$ EDTA, $10 \mathrm{mM}$ acetylCoA, $10 \mathrm{mM}$ DTNB, $\mathrm{pH}$ 8.0) and measured the reduction of DTNB at $412 \mathrm{~nm}$ with a Tecan-Infinite ${ }^{\circ} 200$ Pro (Tecan Group Ltd., Männedorf, Switzerland). To measure HMGR activity, crude latex harvested from 8-weekold roots of T. brevicorniculatum plants was transferred to ice-cold rubber extraction buffer, and $\sim 40 \mu \mathrm{g}$ total protein was used in a $\left[3-{ }^{14} \mathrm{C}\right] \mathrm{HMG}-\mathrm{CoA}$ incorporation assay as previously described $[32,35]$.

\section{Staining root cross-sections with Nile red}

Taraxacum brevicorniculatum roots were sliced with a razor blade and incubated in a Nile red staining solution ( $1 \mathrm{mg} / \mathrm{ml}$ Nile red in dimethylsulfoxide (DMSO) stock solution diluted 1:150 in double-distilled water) for $30 \mathrm{~min}$. Stained root cross-sections were analyzed under a Leica MZ16 F fluorescence stereomicroscope (Leica Microsystems GmbH, Wetzlar, Germany) using the GFP Plants filter set (excitation filter $450-490 \mathrm{~nm}$, barrier filter $500-550 \mathrm{~nm}$ ) and visualized with Leica Application Suite X software.

\section{Chemical analysis}

Triterpene and poly(cis-1,4-isoprene) levels from T. brevicorniculatum root material were quantified via gas chromatography/mass spectrometry (GC/MS) and ${ }^{1} \mathrm{H}$ nuclear magnetic resonance spectroscopy $\left({ }^{1} \mathrm{H}-\mathrm{NMR}\right)$, respectively, as previously described [36]. Dolichol levels were determined as previously described [5] using 1-galiquots of freeze-dried and ground T. brevicorniculatum root material.

\section{Statistical analysis}

Normal distributions of gene expression levels and enzymatic activities at $p<0.05$ were assessed using the Kolmogorov-Smirnov test in OriginPro 2016 (OriginLab, Northhampton, USA). Normally distributed datasets were analyzed using ANOVA with the post-hoc Tukey's honest significant difference test. Datasets which did not show a normal distribution and could not be transformed to be normally distributed were analyzed using the Mann-Whitney U test.

\section{Results and discussion}

Synthesis of the full-length $A C L A, A C L B$ and $A A C T$ CDNA sequences from $T$. brevicorniculatum latex RNA

The T. brevicorniculatum genome has not yet been sequenced, so expressed sequence tag (EST) databases at GenBank prepared from either root material of its close relative $T$. koksaghyz or from $T$. officinale (tissue not specified at GenBank) were searched for ACLA, ACLB and $A A C T$ cDNA sequences. Two different T. koksaghyz 
ACLA ESTs (GenBank accession numbers DR401254 and DR402930) as well as one T. officinale ACLA EST (DY808625) were found which covered the $5^{\prime}$ end of $A C L A$ open reading frames based on comparisons with the A. thaliana ACLA genes At1g10670, At1g60810 and At1g09430. One full-length cDNA sequence could be amplified by 3'RACE using $T$. brevicorniculatum root latex RNA as template. The 1272-bp TbACLA1 cDNA (GenBank accession number KY765686) based on the overlapping ESTs DR401254 and DR402930 encoded a protein of 423 amino acids. Furthermore, two different $T$. koksaghyz ESTs (DR398498 and DR399293) were found with high similarity to the $A$. thaliana $A C L B$ orthologs At3g06650 and At5g4946. By performing 5'RACE using the same template one full-length TbACLB cDNA (1827 bp, GenBank accession number KY765687) encoding a protein of 608 amino acids named TbACLB1 (based on EST DR398498) was produced. Finally, T. koksaghyz ESTs DR398619 and DR401218 showed high similarity to the A. thaliana AACT genes At5g47720 and At5g48230. One full-length cDNA was produced by 3 'RACE again using T. brevicorniculatum root latex RNA as the template. The 1227-bp TbAACT1 cDNA sequence (GenBank accession number KY765685) based on EST DR398619 encoded a protein of 408 amino acids.

The deduced polypeptides of full-length cDNAs were aligned in silico with known ACLs and AACTs from $A$. thaliana, Oryza sativa and $H$. brasiliensis using Clustal Omega (https://www.ebi.ac.uk/Tools/msa/clustalo/) [see Additional file 3]. The TbACLA1 amino acid sequence showed $79-85 \%$ identity to ACLAs from A. thaliana (AtACLA1-3; Q9SGY2.1, O22718.1 and O80526.1) and O. sativa (OsACLA1-3; Q53JY8.2, Q2QZ86.2 and Q2QNG7.1). As shown in Additional file 3 A, TbACLA1 contains the ACL-SCS family signature 3 motif (PROSITE accession number PS01217) at residues 270-294 as well as the residues Lys4, Lys58, Glu116 and Asp213, which are conserved in the ATP grasp domain [23, 24].

The TbACLB1 amino acid sequence showed $90-91 \%$ identity to AtACLB1 (Q9C522.1), AtACLB2 (Q9FGX1.1) and OsACLB1 (Q93VT8.1). The multiple sequence alignment suggested the presence of the above mentioned ACL-SCS family signature 3 motif at TbACLB1 residues 168-193, as well as the ACL-SCS family signature 1 motif (PROSITE accession number PS01216) at TbACLB1 residues 174-203 and the ACL-SCS family active site (PROSITE accession number PS00399) at TbACLB1 residues 259-275, including the His273 residue which is thought be phosphorylated by ATP [see Additional file $3 \mathrm{~B}$ ]. This confirmed the homology between TbACLB1 and SCS $\alpha$-subunits.

The TbAACT1 protein sequence showed $79 \%$ identity to HbAACT1 (BAF98276.1), 78\% identity to AtAACT2 (NP_568694.2) and 75\% identity to
AtAACT1 (NP_199583.1). The multiple sequence alignment indicated the presence of thiolase signature 2 (PROSITE accession number PS00737) at TbAACT1 residues 354-370, and the thiolase active site (PROSITE accession number PS00099) at TbAACT1 residues 389-402, including residues involved in the thiolase reaction cycle [37] [see Additional file $3 \mathrm{C}$ ]. The greater sequence identity between TbAACT1 and AtAACT2 compared to the functionally redundant AtAACT1 [28] suggests that TbAACT1 is the dominant AACT isoform in $T$. brevicorniculatum.

\section{Transcriptional regulation of the $T b A C L A, T b A C L B$ and TbAACT genes}

To determine whether the isolated TbACLA1, TbACLB1 and TbAACT1 genes encoded the dominant isoforms in the latex, qRT-PCR analyses were performed using RNA from the latex, root, peduncle, leaf and flower tissues of 10-week-old wild-type T. brevicorniculatum plants grown under greenhouse conditions. Furthermore, qRT-PCR primer pairs were included in the analysis that were based on the additional ESTs mentioned above which are partial cDNAs encoding a second isoform of ACLA, ACLB and AACT, named TbACLA2 (based on DY808625), TbACLB2 (DR399293) and TbAACT2 (DR401218), respectively. For comparison, parallel qRT-PCR tests were carried out on the TbHMGR1 and TbHMGR2 genes, whose expression profiles have already been determined by RT-PCR [19]. TbHMGR1 plays a pivotal role in the latex MVA pathway due to its expression predominantly in laticifers, whereas TbHMGR2 expression is barely detected in latex but it is expressed more strongly than TbHMGR1 in roots and leaves [19] [see Additional file 4]. Like TbHMGR1, we found that TbACLA1, TbACLB1 and TbAACT1 were predominantly expressed in the latex [see Fig. $2 \mathrm{a}-\mathrm{c}$ ]. The expression of these genes detected in all other tissues may reflect the presence of small amounts of latex in all parts of the dandelion plant, but might also reflect the necessity of a basal activity of theses enzymes to synthesize important MVA pathway-derived substances for regular plant development. Remarkably, an overall lower expression could be measured for TbACLB2 and TbAACT2 in all tissues analyzed compared to $T b A C L B 1$ and $T b A A C T 1$, respectively, whereas $T b A C L A 2$ showed a similar expression level in roots and even slightly increased expression levels in peduncle, leaf and flower tissues (3-4 fold) compared to TbACLA1 indicating that TbACLA2 is an important isoform in these tissues. However, the tissue-specific qRTPCR analyses clearly demonstrate that TbACLA1, TbACLB1 and TbAACT1 represent the dominant isoforms in latex and therefore appear to be highly relevant components of the T. brevicorniculatum latex MVA pathway and are likely to be required for the production of isoprenoid end products in this specialized tissue. 


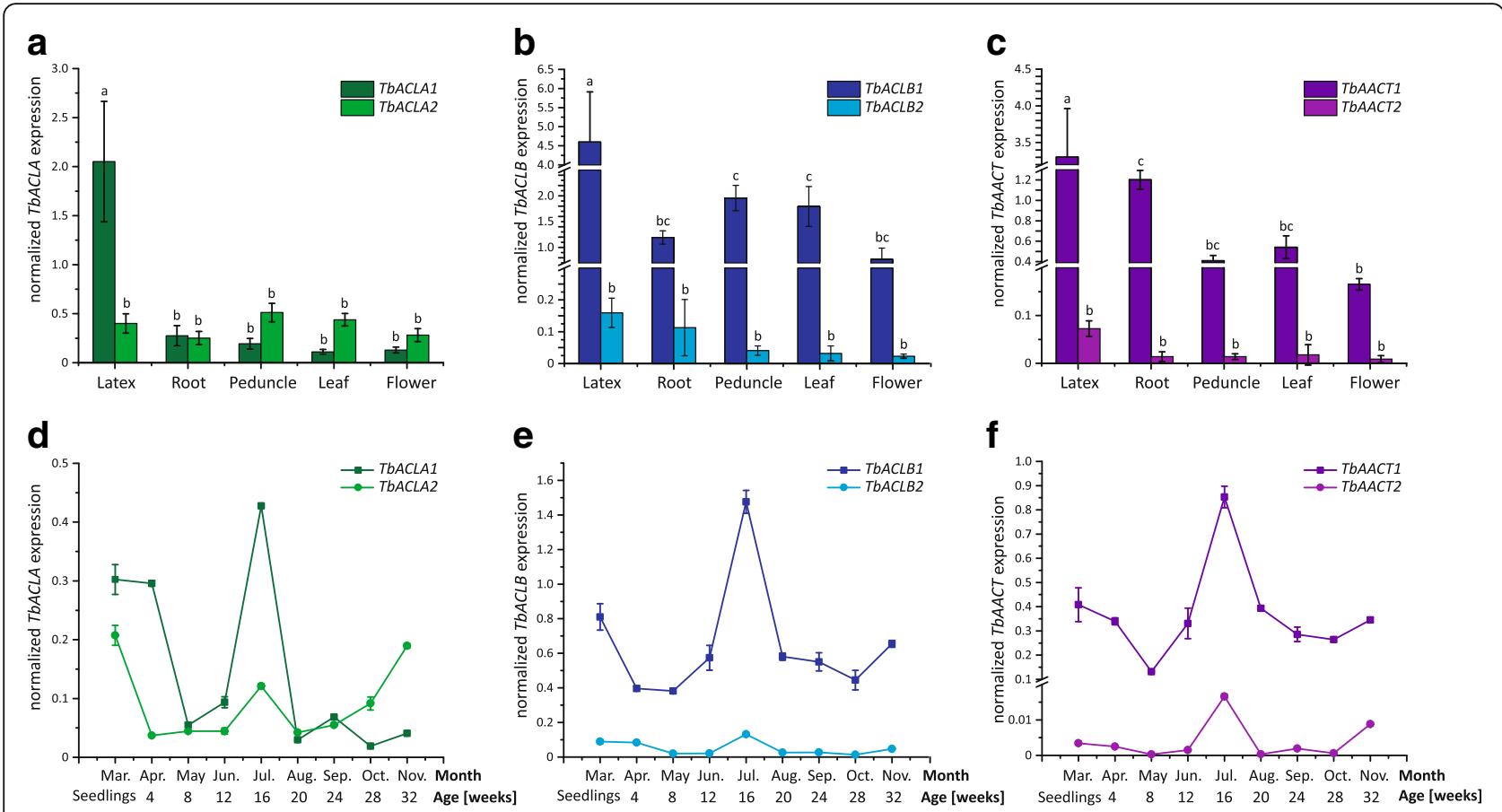

Fig. 2 Spatial and temporal ACLABB and AACT mRNA expression profile in wild-type T. brevicorniculatum plants determined by qRT-PCR. The corresponding mRNA levels were normalized against the constitutive genes elongation factor 1 a (TbEF1a), glyceraldehyde-3-phosphate dehydrogenase (TbGAPDH) and ribosomal protein L27 (TbRP) from T. brevicorniculatum. Bars represent standard errors of three independent wild-type plants per line. Normal distribution at $p<0.05$ was assessed using the Kolmogorov-Smirnov test. Different letters indicate a significant difference (ANOVA with Tukey's honest significant difference test $(p<0.01)$ ). a TbACLA1 and TbACLA2 b TbACLB1 and TbACLB2 and $\mathbf{c}$ TbAACT1 and TbAACT2 mRNA levels in latex, roots, peduncles, leaves and flowers of 10-week-old wild-type T. brevicorniculatum plants. d TbACLA1 and TbACLA2, e TbACLB1 and TbACLB2 and f TbAACT1 and TbAACT2 mRNA levels in seedlings and root material of wild-type T. brevicorniculatum plants ranging in age from 4 to 32 weeks. Plants flowered in June

The potential co-regulated expression of $T b A C L A$, $T b A C L B$ and TbAACT during dandelion development was investigated by cultivating $T$. brevicorniculatum wild-type plants outside the greenhouse from March to November. Total root material (containing the greatest amount of latex in relation to other tissues) was pooled from three individual plants per month and tested by qRT-PCR [see Fig. 2d-f]. In accordance to the tissue expression analysis, TbACLB1 and TbAACT1 comprised higher expression levels over the whole year in the dandelion root compared to TbACLB2 and TbAACT2, respectively [see Fig. 2e and $\mathrm{f}$ ], whereas in case of TbACLA the isoform TbACLA2 seemed to play an important role at defined developmental stages such as in October and November [see Fig. 2d]. Overall, TbACLA, $T b A C L B$ and TbAACT displayed highly similar expression patterns with expression peaks at the seedling stage in March, and also in July and November indicative of tight co-regulation, as already reported for the $A$. thaliana ACLA and ACLB subunits [24]. Given that $A$. thaliana ACLA and ACLB mRNA accumulates in the epidermis and trichomes of expanding leaves as well as the epidermis of growing organs [24], ACL gene expression seems to be associated with (and upregulated during) plant growth, as supported by the developmental and growth defects observed in $A$. thaliana $A C L-$ RNAi plants [25]. Therefore, ACL activity in the MVA pathway may be pivotal when large quantities of isoprenoids are required, e.g. for the synthesis of membrane sterols during development. The expression peaks in early development and in the summer were coordinated with the upregulation of TbAACT in March and July. This indicated that the $T b A C L$ and $T b A A C T$ genes are coregulated to ensure the shuttling of precursors into the MVA pathway during these developmental stages which are characterized by rapid expansion of the total root volume and consequently also the differentiation of the specialized laticiferous cells. Interestingly, $T b A C L$ and TbAACT showed an additional expression peak in November, which is when dandelion plants accumulate high levels of polyisoprenes in the roots [36]. This might explain the highly active MVA pathway in the latex and therefore the additional upregulation of TbACL for providing precursors and TbAACT as the first enzymatic step in the cytosolic isoprenoid pathway.

Gene expression levels do not necessarily reflect the levels of AACT and HMGR enzyme activity due to downstream regulatory mechanisms such as phosphorylation, feedback inhibition and external stimuli [16, 17, 29, 38, 39]. Therefore, the posttranscriptional regulation 
of key MVA pathway enzymes will be an important additional aspect to address in future studies concerning the engineering of isoprenoid metabolism in dandelion latex.

\section{Overexpression of ACL, AACT and HMGR orthologs from A. thaliana in dandelion latex}

Having identified and characterized the T. brevicorniculatum latex-specific isoforms of ACL and AACT, we investigated the effects of overexpressing these enzymes, using the better-characterized $A$. thaliana orthologs to reduce the likelihood of endogenous gene inhibition. We previously reported that overexpressing the $A$. thaliana ATP citrate lyase fusion construct $\operatorname{AtACL}(A B)$ enhanced ACL activity in T. brevicorniculatum [26]. Therefore, a transgenic T. brevicorniculatum line expressing AtA$C L(A B)$ from our previous study [26] was used here and served as a control line to complete the setup. The $A$. thaliana genome contains two paralogous AACT genes (AtAACT1 and AtAACT2) although only AtAACT2 is ubiquitously expressed [28]. The AtAACT2 cDNA sequence was therefore cloned from $A$. thaliana leaf RNA and used for the stable transformation of wild-type $T$. brevicorniculatum plants.

HMGR catalyzes the rate-limiting step in the MVA pathway, and in A. thaliana the AtHMGR1 gene is ubiquitously expressed [40]. The catalytic activity of plant HMGRs is inhibited by the $\mathrm{N}$-terminal domain and by phosphorylation of the C-terminus, so we tested the activity of two different variants of AtHMGR1 in the heterologous $N$. benthamiana system. Athmgrc1(S408A) comprises the catalytic domain including the mutated phosphorylation site at position 408 , and this was 2.5 -fold more active than the wild-type catalytic domain Athmgrc1 [see Additional file 5]. Athmgrc1(S408A) was therefore used for the stable transformation of wild-type $T$. brevicorniculatum plants. The potential synergistic effect of $\operatorname{AtACL}(A B)$, AtAACT2 and Athmgrc1(S408A) overexpression was investigated by supertransforming transgenic $T$. brevicorniculatum plants expressing $A t A C L(A B)$ with constructs carrying AtAACT2 and Athmgrc1(S408A). All cDNAs were driven by the rubber elongation factor (REF) promoter to ensure preferential expression in the latex [41]. The constructs prepared for stable transformation are shown in Fig. 3a.

A set of 5-10 transgenic plant lines representing each transformation experiment was characterized by either RT-PCR or qRT-PCR in the T0 generation (data not shown). We then selected one line expressing the AtA$\mathrm{CL}(\mathrm{AB})$ fusion construct (ACL-1), two lines overexpressing AtAACT2 (AACT-3 and AACT-4), two lines expressing Athmgrc1(S408A) (HMGR-7 and HMGR-8) and two lines expressing all three enzymes (ACL-AACTHMGR-4 and ACL-AACT-HMGR-5) for detailed analysis in the $\mathrm{T} 1$ generation. Transgene expression in the latex harvested from roots of 8-week-old plants from all seven transgenic lines was confirmed by qRT-PCR [see Additional file $6 \mathrm{~A}$ ]. The expression levels of the endogenous TbACLA, TbAACT and TbHMGR1 genes were also compared to wild-type plants using the same technique [see Additional file 6 B]. No significant differences in endogenous gene expression levels were observed between wildtype plants and the transgenic lines, indicating that the overexpression of $A$. thaliana genes did not affect endogenous gene expression. We also measured the levels of the corresponding endogenous and heterologous proteins. Latex protein extracts obtained from roots were analyzed by western blot using antibodies specific for each enzyme [see Fig. 3b]. The anti-ACLB antibody revealed the presence of a $66 \mathrm{kDa}$ band corresponding to the endogenous subunit in all lines and the presence of a $120 \mathrm{kDa}$ band corresponding to the chimeric AtACL(AB) protein solely in the transgenic lines expressing the AtACL(AB) construct (ACL-1, ACL-AACT-HMGR-4 and ACL-AACTHMGR-5). Likewise, the anti-AtAACT2 antibody revealed two protein bands of $\sim 43 \mathrm{kDa}$ and $\sim 41 \mathrm{kDa}$, with the upper band corresponding to the endogenous TbAACT protein and the lower band corresponding to the AtAACT2 protein (the latter only present in transgenic lines overexpressing AACT: AACT-3, AACT-4, ACLAACT-HMGR-4 and ACL-AACT-HMGR-5). Finally, the anti-HMGR antibody revealed one protein band of $\sim 63 \mathrm{kDa}$ representing the predominant endogenous latex isoform (TbHMGR1) and another of $\sim 45 \mathrm{kDa}$ representing the catalytic domain Athmgrc1(S408A), the latter present only in transgenic lines HMGR-7, HMGR-8, ACL-AACT-HMGR-4 and ACL-AACTHMGR-5. These data confirmed that all transgenes were expressed in the latex as anticipated and were translated into the corresponding proteins without affecting endogenous protein levels.

Having confirmed the presence of the heterologous proteins and the absence of effects on endogenous proteins, we next measured the corresponding enzyme activities in latex crude protein extracts obtained from roots by determination of the conversion of $\left[1,5-{ }^{14} \mathrm{C}\right]$ citric acid to ${ }^{14} \mathrm{C}$-labeled acetyl-CoA (ACL), the reduction of DTNB by free CoA (AACT) and the conversion of $\left[3-{ }^{14} \mathrm{C}\right]$ HMG-CoA to $\left[{ }^{14} \mathrm{C}\right]$ mevalonolactone (HMGR) [see Fig. 3c]. ACL activity was significantly higher in the three transgenic lines expressing AtACL(AB) than the other lines (3.3-fold, 3.1-fold and 3.1-fold increases in lines ACL-1, ACL-AACT-HMGR4 and ACL-AACT-HMGR-5, respectively). AACT activity was slightly higher in the four transgenic lines expressing the AtAACT2 construct (1.3-fold, 1.4-fold, 1.3-fold and 1.2-fold increases in lines AACT-3, AACT-4, ACL-AACTHMGR4 and ACL-AACT-HMGR-5, respectively). Finally, all four transgenic lines expressing Athmgrc1(S408A) showed significantly higher levels of HMGR activity than the other lines (4.0-fold, 3.4-fold, 5.1-fold and 4.6-fold 


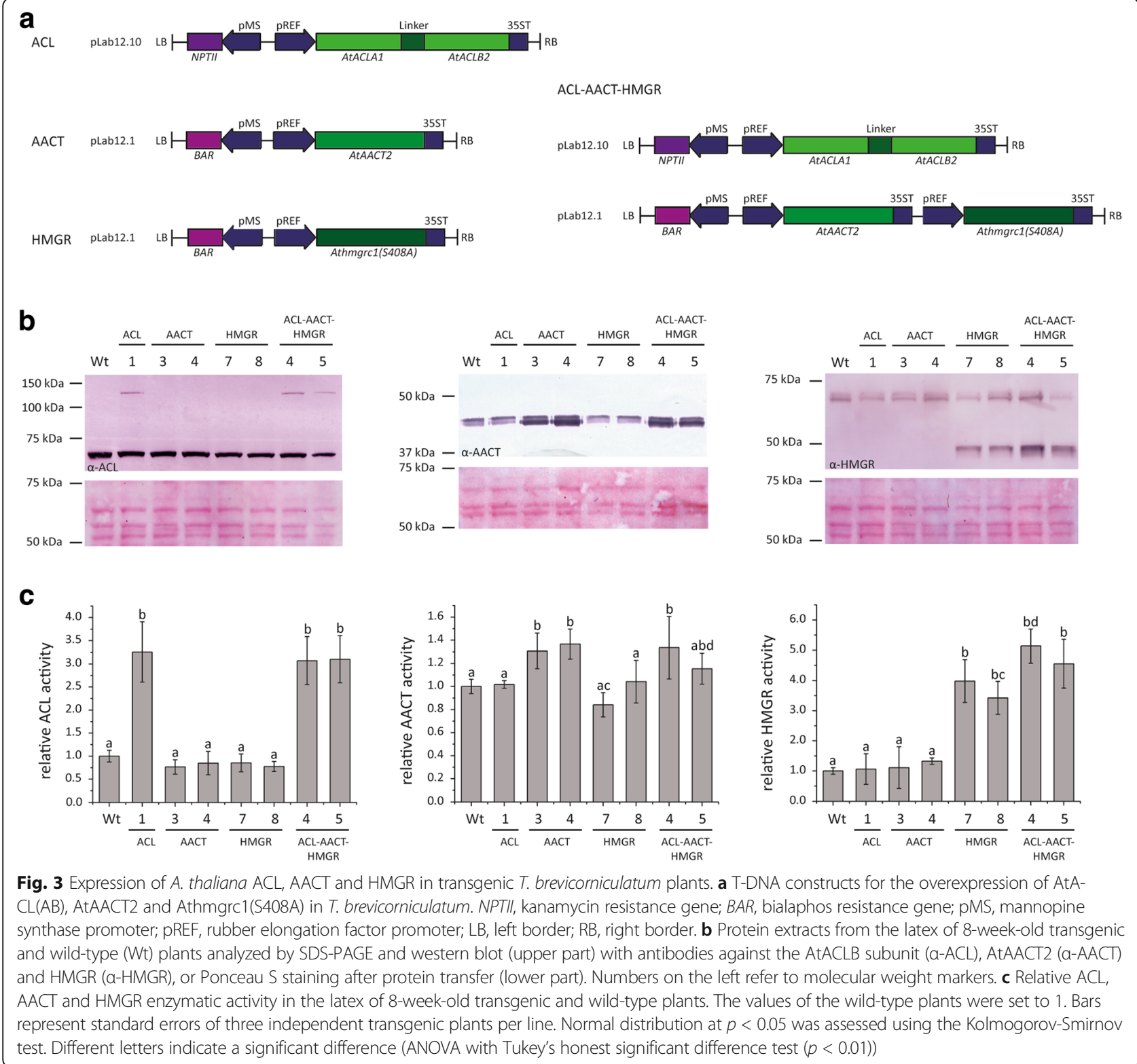

increases in lines HMGR-7, HMGR-8, ACL-AACTHMGR-4 and ACL-AACT-HMGR-5, respectively). To our knowledge, this is the first report of the successful simultaneous overexpression of three functional transgenes in the latex of T. brevicorniculatum.

\section{Upregulation of the MVA pathway in T. brevicorniculatum latex enhances the accumulation of squalene, sterols, pentacyclic triterpenes and cis-1,4-isoprene}

Whole roots from three 12-week-old plants per line were harvested and tested for their phenotypic and metabolic characteristics. The transgenic lines showed no overt phenotypic aberrations (representative images of one plant per line are compared to a wild-type plant in Additional file 7 A). Furthermore, there were no significant differences between the wild-type plants and transgenic lines in terms of root fresh weight or root dry weight [see Additional file $7 \mathrm{~B}$ and $\mathrm{C}]$. The overexpression of heterologous AtACL(AB), AtAACT2 and Athmgrc1(S408A) in the latex therefore had no significant impact on the growth or development of T. brevicorniculatum. To ensure no major costs arise from this overexpression of transgenes, it would be necessary to measure the overall plant fitness by e.g. evaluating the seed production, quality and quantity.

Freeze-dried roots were used for triterpene extraction and GC/MS analysis because the quantitative analysis of triterpenes in latex tissue as described in Huber et al. [42] was not feasible for comparative analysis of wild-type and transgenic plant lines. The quantity of triterpene precursors 
was considerably higher in transgenic lines HMGR-7, HMGR-8, ACL-AACT-HMGR-4 and ACL-AACTHMGR-5 than the other lines [see Fig. 4a]. Although the levels of the precursor 2,3-oxidosqualene were only slightly (1.7-1.9-fold) higher in the transgenic lines compared to wild-type plants, large amounts of squalene accumulated in the roots of the triple-transgenic lines (up to $32 \mathrm{mg} / \mathrm{g}$ root dry weight). Squalene accumulation was therefore investigated morphologically in cross-sections of wild-type and transgenic roots [see Additional file 8]. Nile red, which stains lipophilic molecules, revealed the presence of globules resembling lipid droplets in the root sections of all four transgenic lines. These globules were much more abundant in lines ACL-AACT-HMGR-4 and ACL-AACT-HMGR-5 than lines HMGR-7 and HMGR-8, and differed from laticifers in terms of size and position. These data suggest that excess squalene produced in these four transgenic lines is stored in lipid droplets outside the laticifers. These findings are supported by the observation of similar lipid droplets in tobacco

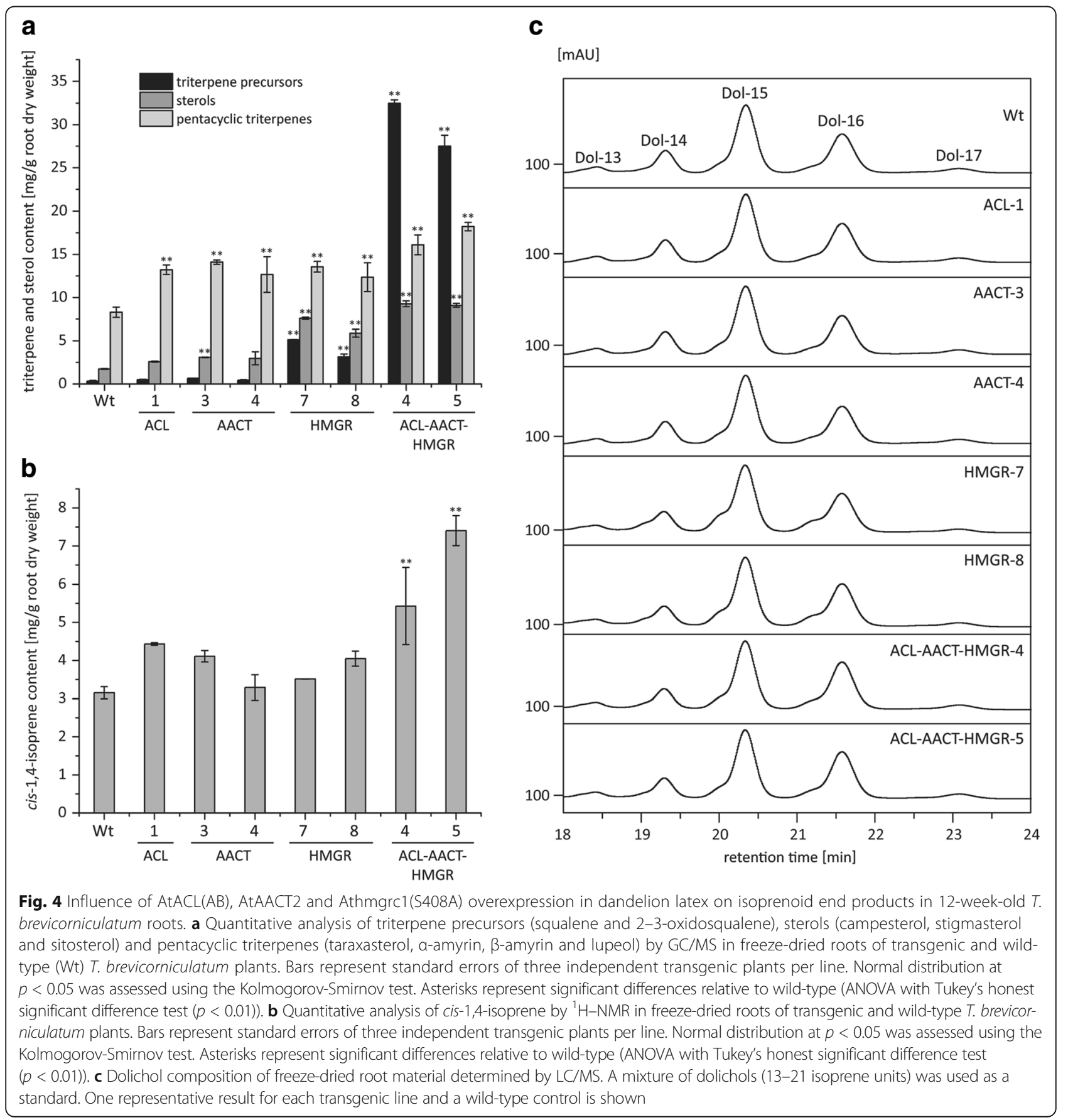


(Nicotiana tabacum) leaves expressing full-length or truncated HbHMGR1 [10, 15].

The average quantity of squalene accumulating in the roots of the triple-transgenic lines was $31.8 \mathrm{mg} / \mathrm{g}$ dry root weight for line ACL-AACT-HMGR-4 and $26.95 \mathrm{mg} / \mathrm{g}$ dry root weight for line ACL-AACT-HMGR-5. With the exception of a genetically engineered strain of Pseudozyma sp. $(70 \mathrm{mg} / \mathrm{g}$ dry cell weight (DCW)) [43], this exceeds the highest squalene yields thus far achieved in microbes such as Saccharomyces cerevisiae (18.5 mg/g DCW), Rhodopseudomonas palustris (15.8 mg/g DCW) and Schizochytrium mangrovei $(1.17 \mathrm{mg} / \mathrm{g} \mathrm{DCW})$ [44-46], but is lower than in plant tissues such as Amaranthus spp. seed oil $(7 \% \mathrm{w} / \mathrm{w})$ and olive oil $(30 \% \mathrm{w} / \mathrm{w})[47,48]$. However, the genetically engineered squalene content in transgenic T. brevicorniculatum roots by simultaneous overexpression of $\mathrm{ACL}$, AACT and HMGR is within an industrially relevant range. Interestingly, the large amount of squalene present in the lipid droplets in the T. brevicorniculatum root does not appear to be toxic, which is consistent with experiments in S. cerevisiae showing that squalene accumulation is toxic only when lipid droplet formation is impaired [49]. The similar levels of 2,3-oxidosqualene in wild-type and transgenic plants despite the substantial differences in squalene levels indicate that the formation of 2,3-oxidosqualene catalyzed by squalene epoxidase might act as the main bottleneck between the MVA pathway and downstream isoprenoid biosynthesis.

We also analyzed the levels of other sterols, such as campesterol, stigmasterol and sitosterol. Overall, the quantity of sterols was higher in the roots of the transgenic lines than wild-type plants but the extent of sterol accumulation was dependent on the complement of transgenes. In the lines expressing $\mathrm{ACL}$ or AACT, the sterol levels were less than 2-fold higher than wild-type levels. In contrast, the sterol levels were 4.2-fold, 3.3fold, 5.2-fold and 5.1-fold higher than wild-type in transgenic lines HMGR-7, HMGR-8, ACL-AACT-HMGR-4 and ACL-AACT-HMGR-5, respectively. This shows that the overexpression of ACL, AACT and HMGR increased flux through the MVA pathway in terms of IPP units directed towards sterol biosynthesis. Transgenic T. brevicorniculatum lines expressing HMGR produced the highest quantity of sterols, confirming that HMGR is the pivotal rate-limiting enzyme in the MVA pathway. Similarly, the overexpression of HbHMGR1 in tobacco achieved a 6-fold increase in sterol levels [10] and the seed-specific expression of a truncated HbHMGR1 led to a 3.2-fold increase in seed sterol accumulation [15]. Our novel approach involved the simultaneous expression of three enzymes involved in the MVA pathway, achieving even higher sterol levels than the expression of HMGR alone.
Pentacyclic triterpenes such as taraxasterol, $\alpha$-amyrin, $\beta$ amyrin and lupeol are also important triterpene components of $T$. brevicorniculatum latex because of their potential medical applications. For example, $\alpha$-amyrin from Bombax malabaricum flowers is active against a range of bacteria and fungi [50] whereas $\alpha$-amyrin, $\beta$-amyrin and lupeol display anti-inflammatory activities in mice and mammalian cell lines [51-53]. Taraxasterol has antiallergic, anti-inflammatory and anti-carcinogenic effects [54-57]. These compounds are therefore valuable products in the biotechnology industry and efforts have already been undertaken to increase their yields, e.g. the addition of abiotic elicitors to root callus suspension cultures of $T$. officinale, a close relative of $T$. brevicorniculatum, to increase the production of taraxasterol [58]. In our transgenic lines overexpressing a single enzyme, the pentacyclic triterpene content increased by 1.5-1.7-fold compared to wild-type plants. In the triple-transgenic lines ACL-AACT-HMGR-4 and ACL-AACT-HMGR-5, the corresponding increase was 1.9-2.2-fold, suggesting that the overexpression of three MVA pathway enzymes has a synergistic effect. Such effects could therefore enhance the industrial production of pentacyclic triterpenes, especially in combination with the metabolic engineering of downstream pathways.

Next, we set out to determine the total cis-1,4-isoprene content in $T$. brevicorniculatum roots by ${ }^{1} \mathrm{H}-\mathrm{NMR}$ spectroscopy, which includes the analysis of polyisoprenes such as dolichols as well as high-molecular-weight long-chain poly(cis-1,4-isoprenes) that form the main constituent of natural rubber [see Fig. 4b]. All transgenic lines accumulated similar or slightly higher levels of cis-1,4-isoprene compared to wild-type plants, with lines ACL-1, ACLAACT-HMGR-4 and ACL-AACT-HMGR-5 producing the highest levels (1.4-fold, 1.7-fold and 2.3-fold higher than wild-type, respectively). In these transgenic lines, IPP generated by the increase in flux through the MVA pathway was at least in part incorporated into cis-1,4-isoprene end products. We also investigated whether the greater abundance of precursors affected the chain length of known dolichols by liquid chromatography/mass spectrometry (LC/MS). However, no substantial differences in dolichol composition were observed between the wild-type and transgenic lines [see Fig. 4c].

The synthesis of polyisoprenes in the transgenic lines was not substantially affected by the overexpression of three key MVA pathway enzymes, which may reflect the bottleneck at the squalene epoxidase step. This step is therefore ideal for more detailed characterization in future experiments. Furthermore, the production of natural rubber in laticifers might not be dependent solely on the availability of IPP units, but also on the presence and activity of the enzymes that are directly linked to the rubber particles and thus build the rubber transferase complex [5]. These specialized end-product enzymes also represent 
useful targets for future genetic engineering approaches to increase the production of isoprenoid end products.

\section{Conclusions}

We isolated full-length $A C L$ and $A A C T$ cDNAs that are involved in production of isoprenoids in $T$. brevicorniculatum latex, namely $T b A C L A, T b A C L B$ and $T b A A C T$. The expression of the corresponding $A$. thaliana genes in T. brevicorniculatum latex, in addition to a derepressed version of HMGR (the rate-limiting enzyme in the MVA pathway) increased the activity of all three enzymes and affected the accumulation of isoprenoid end products. The heterologous overexpression of all three enzymes resulted in the accumulation of large amounts of squalene as well as higher levels of sterols, pentacyclic triterpenes and cis-1,4-isoprene in the roots of the transgenic lines. The synergistic effect of the three key enzymes could facilitate the industrial production of squalene or triterpenes. The yield could be improved substantially, especially in combination with the metabolic engineering of downstream enzymes.

\section{Additional files}

Additional file 1: Sequences of oligonucleotides used for cloning and qRT-PCR. (PDF $81 \mathrm{~kb}$ )

Additional file 2: Primer efficiency, amplification factors and formamide usage for cDNA obtained from T. brevicorniculatum mRNA. The values were calculated using the Bio-Rad CFX Manager 3.1 software (Bio-Rad Laboratories Inc., Hercules, USA) and the qPCR primer efficiency calculator provided by Thermo Fisher Scientific (http://www.thermoscientificbio.com/webtools/qpcrefficiency/). (PDF $60 \mathrm{~kb}$ )

Additional file 3: In silico analysis of TbACLA1, TbACLB1 and TbAACT1 from T. brevicorniculatum. Alignments were created with Clustal Omega (https://www.ebi.ac.uk/Tools/msa/clustalo/). A: ACLA amino acid alignment with protein sequences from $A$. thaliana (At), O. sativa (Os) and T. brevicorniculatum (Tb). Sequences for AtACLA1 (Q9SGY2.1), AtACLA2 (O22718.1), AtACLA3 (O80526.1), OsACLA1 (Q53JY8.2), OsACLA2 (Q2QZ86.2) and OsACLA3 (Q2QNG7.1) were obtained from GenBank (https://www.ncbi.nlm.nih.gov/genbank). Conserved residues representing the ATP grasp domain (Lys4, Lys48, Glu116 and Asp213) are shaded in light blue, and the ACL-SCS family signature 3 motif (PROSITE accession number PS01217) is shown in the yellow box. B: ACLB amino acid alignment with protein sequences from $A$. thaliana (At), O. sativa (Os) and T. brevicorniculatum (Tb). Sequences for AtACLB1 (Q9C522.1), AtACLB2 (Q9FGX1.1) and OsACLB1 (Q93VT8.1) were obtained from GenBank (https://www.ncbi.nlm.nih.gov/genbank/). The ACL-SCS family signature 3 motif (PROSITE accession number PS01217) and ACL-SCS signature 1 motif (PROSITE accession number PS01216) are shown in the green box, whereas the ACL-SCS family active site (PROSITE accession number PS00399) is shown in the pink box with the conserved (phosphorylated) residue His273 highlighted in light blue. C: AACT amino acid alignment with protein sequences from $A$. thaliana $(A t), H$. brasiliensis $(\mathrm{Hb})$ and $T$. brevicorniculatum (Tb). Sequences for AtAACT1 (NP_199583.1), AtAACT2 (NP_568694.2) and HbAACT1 (BAF98276.1) were obtained from GenBank (https://www.ncbi.nlm.nih.gov/genbank/). Conserved sites included thiolase signature 2 (PROSITE accession number PS00737, yellow box) and thiolase active site (PROSITE accession number PS00099, pink box). Residues involved in the thiolase reaction cycle are indicated in green [37]. (PDF $61 \mathrm{~kb}$ )

Additional file 4: Spatial HMGR expression profile in 10-week-old wildtype T. brevicorniculatum plants. Normalized HMGR1 and HMGR2 mRNA levels in latex, roots, peduncles, leaves and flowers were determined by
qRT-PCR. The corresponding mRNA levels were normalized against the constitutive genes elongation factor 1 a (TbEF1a), glyceraldehyde-3phosphate dehydrogenase (TbGAPDH) and ribosomal protein L27 (TbRP) from T. brevicorniculatum. Bars represent standard errors ( $n=$ three biological replicates). Normal distribution at $p<0.05$ was assessed using the Kolmogorov-Smirnov test. Different letters indicate a significant difference (ANOVA with Tukey's honest significant difference test $(p<0.01)$ ). (TIFF $187 \mathrm{~kb}$ )

Additional file 5: Relative HMGR activity of two different Athmgrc1 variants in the heterologous $N$. benthamiana system. A: HMGR activity measured in $N$. benthamiana leaf extracts following the transient expression of Athmgrc1 constructs for 1 week. Athmgrc1, catalytic domain of AtHMGR1; Athmgrc1(S408A), catalytic domain of AtHMGR1 with a serine to alanine substitution at position 408. B: Protein extracts from $N$. benthamiana leaves transiently expressing Athmgrc1 variants analyzed by SDS-PAGE and western blot (upper part) using an antibody against HMGR. Ponceau $\mathrm{S}$ staining after protein transfer is shown below. Numbers on the left refer to molecular weight markers. (TIFF $116 \mathrm{~kb}$ )

Additional file 6: Normalized ACL, AACT and HMGR mRNA levels in transgenic and wild-type T. brevicorniculatum plants quantified by qRT-PCR. The corresponding mRNA levels were normalized against the constitutive gene elongation factor 1 a (TbEF1a) from T. brevicorniculatum. Bars represent standard errors ( $n=$ three biological replicates). A: AtACLA1, AtAACT2 and AtHMGR1 transgene mRNA levels in transgenic lines. No significant differences at $p<0.05$ were detected among the transgenic lines using the MannWhitney $U$ test. B: Endogenous TbACLA, TbAACT and TbHMGR1 mRNA levels in all transgenic lines and wild-type $(\mathrm{Wt})$ plants. No significant differences at $p<0.05$ were detected between the wild-type plants and transgenic lines using the Mann-Whitney $U$ test. (TIFF $1510 \mathrm{~kb}$ )

Additional file 7: Root morphology and weight of 12-week-old wildtype and transgenic T. brevicorniculatum plants. A: Cleaned roots were harvested and one representative plant from each line was photographed. Scale bar: $10 \mathrm{~cm}$. B: Mean root fresh weight and C: mean root dry weight of harvested roots. Bars represent standard errors ( $n=$ three plants; asterisks indicate $n=$ two plants). No significant differences at $p<0.05$ were detected between wild-type $(\mathrm{Wt})$ plants and transgenic lines using the MannWhitney U test. (TIFF $3459 \mathrm{~kb}$ )

Additional file 8: Root cross-sections of 12-week-old wild-type and transgenic T. brevicorniculatum plants. Staining was carried out with Nile red and one representative cross-section is shown for each line. Wt, wildtype; La, laticifer; Ld, lipid droplet. Scale bar: 250 m.. (TIFF 5280 kb)

\section{Abbreviations}

${ }^{1} \mathrm{H}-\mathrm{NMR}:{ }^{1} \mathrm{H}$ nuclear magnetic resonance spectroscopy; AACT: AcetoacetylCoA thiolase; ACL: ATP citrate lyase; At: Arabidopsis thaliana; CS: Citrate synthase; DCW: Dry cell weight; DMSO: Dimethylsulfoxide; DTNB: 5,5'dithiobis-(2-nitrobenzoic acid); DTT: Dithiothreitol; EF1a: Elongation factor-1 alpha; EST: Expressed sequence tag; GAPDH: Glyceraldehyde-3-phosphate dehydrogenase; GC/MS: Gas chromatography/mass spectrometry; Hb: Hevea brasiliensis; HMGR: 3-hydroxy-methyl-glutaryl-CoA reductase; IPP: Isopentenyl pyrophosphate; LAB: Nicotiana benthamiana laboratory isolate; LC/MS: Liquid chromatography/mass spectrometry; Ms.: Medicago sativa; MVA: Mevalonate; Os: Oryza sativa; qRT-PCR: Quantitative RT-PCR; REF: Rubber elongation factor; RP: Ribosomal protein L27; SCS: Succinyl-CoA synthetase; Tb: Taraxacum brevicorniculatum; Wt: Wild-type

\section{Acknowledgements}

We gratefully acknowledge the technical assistance of Daniela Ahlert (University of Muenster, Germany). We also thank Yves Poirier (Department of Plant Molecular Biology, Université de Lausanne, Switzerland), Basil Nikolau (Roy J. Carver Department of Biochemistry, Biophysics and Molecular Biology, lowa State University, USA) and Hubert Schaller (Insitut de Biologie Moléculaire des Plantes, Université de Strasbourg, France) for providing the antibodies used in this study.

\section{Funding}

This work was supported by a grant from the Federal Ministry of Food and Agriculture (FKZ 22002212) and the Federal Ministry of Science and Education of Germany (grant no. FKZ 031A285A). 


\section{Availability of data and materials}

All data generated or analyzed during this study are included in this published article [and its supplementary information files]. The $T$. brevicorniculatum cDNA sequences can be accessed from GenBank (https:// www.ncbi.nlm.nih.gov/genbank/) with the following accession numbers: TbAACT1: KY765685, TbACLA1: KY765686, TbACLB1: KY765687.

\section{Authors' contributions}

K.M.P., N.v.D, K.U. and C.S.G. conceived and designed the experiments. K.M.P., N.v.D. and K.U. performed the experiments. C.S.G. and D.P. analyzed the data. K.M.P., N.vD., C.S.G. and D.P. wrote the paper. All authors read and approved the final manuscript.

\section{Competing interests}

The authors declare that they have no competing interests.

\section{Consent for publication}

Not applicable.

\section{Ethics approval and consent to participate}

Not applicable

\section{Publisher's Note}

Springer Nature remains neutral with regard to jurisdictional claims in published maps and institutional affiliations.

\section{Author details}

'Institute of Plant Biology and Biotechnology, Schlossplatz 8, 48143 Muenster, Germany. ${ }^{2}$ Fraunhofer Institute for Molecular Biology and Applied Ecology (IME), Schlossplatz 8, 48143 Muenster, Germany.

\section{Received: 29 November 2016 Accepted: 10 May 2017}

\section{Published online: 22 May 2017}

\section{References}

1. Hagel JM, Yeung EC, Facchini PJ. Got milk? The secret life of laticifers. Trends Plant Sci. 2008:13:631-9.

2. Schulze Gronover C, Wahler D, Prüfer D. Natural Rubber Biosynthesis and Physic-Chemical Studies on Plant Derived Latex. 2011. p. 75-88.

3. Takahashi S, Lee HJ, Yamashita S, Koyama T. Characterization of cisprenyltransferases from the rubber producing plant Hevea brasiliensis heterologously expressed in yeast and plant cells. Plant Biotechnol. 2012; 29:411-7.

4. Qu Y, Chakrabarty R, Tran HT, Kwon EJG, Kwon M, Nguyen TD, et al. A lettuce (Lactuca sativa) homolog of human Nogo-B receptor interacts with cis-prenyltransferase and is necessary for natural rubber biosynthesis. J Biol Chem. 2015:290:1898-914.

5. Epping J, van Deenen N, Niephaus E, Stolze A, Fricke J, Huber C, et al. A rubber transferase activator is necessary for natural rubber biosynthesis in dandelion. Nat Plants. 2015;1:15048.

6. Hepper CM, Audley BG. The biosynthesis of rubber from B-Hydroxy-Bmethylglutaryl- coenzyme a in Hevea brasiliensis latex. Biochem J. 1969; 114:379-86.

7. Sando T, Takeno S, Watanabe N, Okumoto H, Kuzuyama T, Yamashita A, et al. Cloning and characterization of the 2-C-methyl-D-erythritol 4-phosphate (MEP) pathway genes of a natural-rubber producing plant, Hevea brasiliensis. Biosci Biotechnol Biochem. 2008;72:2903-17.

8. Enjuto M, Lumbreras V, Marín C, Boronat A. Expression of the Arabidopsis HMG2 Gene, encoding 3-Hydroxy-3-Methylglutaryl coenzyme a Reductase, is restricted to Meristematic and floral tissues. Plant Cell. 1995;7:517-27.

9. Lumbreras V, Campos N, Boronat A. The use of an alternative promoter in the Arabidopsis thaliana HMG1 gene generates an mRNA that encodes a novel 3-hydroxy-3-methylglutaryl coenzyme a reductase isoform with an extended N-terminal region. Plant J. 1995;8:541-9.

10. Schaller H, Crausem B, Benveniste P, Chye M, Tan C, Song Y, et al. Expression of the Hevea brasiliensis (H.B.K.) Müll. Arg. 3-Hydroxy-3Methylglutaryl-coenzyme a Reductase 1 in tobacco results in sterol overproduction. Plant Physiol. 1995;109:761-70.

11. Suzuki M, Kamide Y, Nagata N, Seki H, Ohyama K, Kato H. Loss of function of 3-hydroxy-3-methylglutaryl coenzyme A reductase 1 ( HMG1) in
Arabidopsis leads to $\mathrm{dwar}^{\bullet} \mathrm{ng}$, early senescence and male sterility, and reduced sterol levels. Plant J 2004;1:750-761.

12. Liao P, Hemmerlin A, Bach TJ, Chye M-L. The potential of the mevalonate pathway for enhanced isoprenoid production. Biotechnol Adv. 2016;34:697-713.

13. Campos N, Boronat A. Targeting and topology in the membrane of plant 3hydroxy-3-methylglutaryl coenzyme a reductase. Plant Cell. 1995;7:2163-74.

14. Leivar P, González VM, Castel S, Trelease RN, López-Iglesias C, Arró M, et al. Subcellular localization of Arabidopsis 3-Hydroxy-3-Methylgluraryl-coenzyme a Reductase. Plant Physiol. 2005;137:57-69.

15. Harker M, Holmberg N, Clayton JC, Gibbard CL, Wallace AD, Rawlins S, et al. Enhancement of seed phytosterol levels by expression of an $\mathrm{N}$-terminal truncated Hevea brasiliensis (rubber tree) 3-hydroxy-3-methylglutaryl-CoA reductase. Plant Biotechnol J. 2003;1:113-21.

16. Dale S, Becerra B, Morrice NG, Boronat A, Hardie DG, Ferrer A. Bacterial expression of the catalytic domain of 3-hydroxy-3-methylglutaryl-CoA reductase (isoform HMGR1) from Arabidopsis thaliana, and its inactivation by phosphorylation at Ser577 by Brassica oleracea 3-hydroxy-3-methylglutarylCoA reductase kinase. Eur J Biochem. 1995;233:506-13.

17. Leivar P, Antolín-Llovera M, Ferrero S, Closa M, Arró M, Ferrer A, et al. Multilevel control of Arabidopsis 3-hydroxy-3-methylglutaryl coenzyme a reductase by protein phosphatase 2A. Plant Cell. 2011:23:1494-511.

18. Guo D, Yi H-Y, Li H-L, Liu C, Yang Z-P, Peng S-Q. Molecular characterization of HbCZF1, a Hevea brasiliensis CCCH-type zinc finger protein that regulates hmg1. Plant Cell Rep. 2015;34:1569-78.

19. van Deenen N, Bachmann A-L, Schmidt T, Schaller H, Sand J, Prüfer D, et al. Molecular cloning of mevalonate pathway genes from Taraxacum brevicorniculatum and functional characterisation of the key enzyme 3hydroxy-3-methylglutaryl-coenzyme a reductase. Mol Biol Rep. 2012;39: 4337-49.

20. Rodríguez-Concepción M, Boronat A. Breaking new ground in the regulation of the early steps of plant isoprenoid biosynthesis. Curr Opin Plant Biol. 2015;25:17-22.

21. Lange I, Poirier BC, Herron BK, Lange BM. Comprehensive assessment of transcriptional regulation facilitates metabolic engineering of Isoprenoid accumulation in Arabidopsis. Plant Physiol. 2015;169:1595-606.

22. Xing S, Poirier Y. The protein acetylome and the regulation of metabolism. Trends Plant Sci. 2012;17:423-30.

23. Sánchez LB, Galperin MY, Müller M. Acetyl-CoA synthetase from the amitochondriate eukaryote Giardia lamblia belongs to the newly recognized superfamily of acyl-CoA synthetases (nucleoside diphosphate-forming). J Biol Chem. 2000;275:5794-803.

24. Fatland BL, Ke J, Anderson MD, Mentzen WI, Cui LW, Allred CC, et al. Molecular characterization of a heteromeric ATP-citrate lyase that generates cytosolic acetyl-coenzyme a in Arabidopsis. Plant Physiol. 2002;130:740-56.

25. Fatland BL, Nikolau BJ, Wurtele ES. Reverse genetic characterization of Cytosolic acetyl-CoA generation by ATP-citrate Lyase in Arabidopsis. Plant Cell. 2005:17:182-203.

26. Xing $S$, van Deenen $N$, Magliano P, Frahm L, Forestier E, Nawrath C, et al. ATP citrate lyase activity is post-translationally regulated by sink strength and impacts the wax, cutin and rubber biosynthetic pathways. Plant J. 2014; 79:270-84.

27. Dyer JH, Maina A, Gomez ID, Cadet M, Oeljeklaus S, Schiedel AC. Cloning, expression and purification of an acetoacetyl CoA thiolase from sunflower cotyledon. Int J Biol Sci. 2009:5:736-44.

28. Jin $\mathrm{H}$, Song Z, Nikolau BJ. Reverse genetic characterization of two paralogous acetoacetyl CoA thiolase genes in Arabidopsis reveals their importance in plant growth and development. Plant J. 2012;70:1015-32.

29. Soto G, Stritzler M, Lisi C, Alleva K, Pagano ME, Ardila F, et al. AcetoacetylCoA thiolase regulates the mevalonate pathway during abiotic stress adaptation. J Exp Bot. 2011;62:5699-711.

30. Bally J, Nakasugi $\mathrm{K}$, Jia F, Jung $H$, Ho S, Wong M, et al. The extremophile Nicotiana Benthamiana has traded viral defence for early vigour. Nat Plants. 2015;:1:15165

31. Hood E, Gelvin S, Melchers L, Hoekema A. New Agrobacterium helper plasmids for gene transfer to plants. Transgenic Res. 1993;2:208-18.

32. Post J, van Deenen N, Fricke J, Kowalski N, Wurbs D, Schaller H, et al. Laticifer-specific cis-prenyltransferase silencing affects the rubber, triterpene, and inulin content of Taraxacum brevicorniculatum. Plant Physiol. 2012;158: 1406-17.

33. Fricke J, Hillebrand A, Twyman RM, Prufer D, Schulze GC. Abscisic aciddependent regulation of small rubber particle protein Gene expression in 
Taraxacum brevicorniculatum is mediated by TbbZIP1. Plant Cell Physiol. 2013:54:448-64

34. Muoio DM, Noland RC, Kovalik J-P, Seiler SE, Davies MN, DeBalsi KL, et al. Muscle-specific deletion of Carnitine Acetyltransferase compromises glucose tolerance and metabolic flexibility. Cell Metab. 2012;15:764-77.

35. Bach TJ, Lichtenthaler HK. Inhibition by mevinolen of plant growth, sterol formation and pigment accumulation. Physiol Plant. 1983;59:50-60.

36. Stolze A, Wanke A, van Deenen N, Geyer R, Prüfer D, Schulze GC. Development of rubber-enriched dandelion varieties by metabolic engineering of the inulin pathway. Plant Biotechnol J. 2016; doi:10.1111/pbi. 12672.

37. Haapalainen AM, Meriläinen G, Pirilä PL, Kondo N, Fukao T, Wierenga RK. Crystallographic and kinetic studies of human mitochondrial AcetoacetylCOA Thiolase: the importance of potassium and chloride ions for its structure and function. Biochemistry. 2007:46:4305-21.

38. Ruderman S, Kongsawadworakul P, Viboonjun U, Mongkolporn O, Chrestin H. Mitochondrial/Cytosolic acetyl CoA and rubber biosynthesis genes expression in Hevea brasiliensis latex and rubber yield. Kasetsart J (Nat Sci). 2012:46:346-62.

39. Rodríguez-Concepción M, Forés O, Martínez-García JF, González V, Phillips $M A$, Ferrer $A$, et al. Distinct light-mediated pathways regulate the biosynthesis and exchange of Isoprenoid precursors during Arabidopsis seedling development. Plant Cell. 2004;16:144-56.

40. Enjuto M, Balcells L, Campos N, Caelles C, Arró M, Boronat A. Arabidopsis thaliana Contains two differentially expressed 3-hydroxy-3-methylglutarylCoA reductase genes, which encode microsomal forms of the enzyme. Proc Natl Acad Sci U S A. 1994;91:927-31.

41. Laibach N, Hillebrand A, Twyman RM, Prüfer D, Schulze GC. Identification of a Taraxacum brevicorniculatum rubber elongation factor protein that is localized on rubber particles and promotes rubber biosynthesis. Plant $\mathrm{J}$. 2015;82:609-20.

42. Huber M, Triebwasser-Freese D, Reichelt M, Heiling S, Paetz C, Chandran JN, et al. Identification, quantification, spatiotemporal distribution and genetic variation of major latex secondary metabolites in the common dandelion (Taraxacum officinale agg.). Phytochemistry. 2015;115:89-98.

43. Chang $\mathrm{M}-\mathrm{H}$, Kim $\mathrm{H}-\mathrm{J}$, Jahng $\mathrm{K}-\mathrm{Y}$, Hong S-C. The isolation and characterization of Pseudozyma sp. JCC 207, a novel producer of squalene. Appl. Microbiol. Biotechnol. 2008;78:963-72.

44. Mantzouridou F, Tsimidou MZ. Observations on squalene accumulation in Saccharomyces cerevisiae due to the manipulation of HMG2 and ERG6. FEMS Yeast Res. 2010;10:699-707.

45. Xu W, Chai C, Shao L, Yao J, Wang Y. Metabolic engineering of Rhodopseudomonas palustris for squalene production. J Ind Microbiol Biotechnol. 2016;43:719-25

46. Yue C-J, Jiang Y. Impact of methyl jasmonate on squalene biosynthesis in microalga Schizochytrium mangrovei. Process Biochem. 2009;44:923-7.

47. Bondioli P, Mariani C, Lanzani A, Fedeli E, Muller A. Squalene recovery from olive oil deodorizer distillates. J Am Oil Chem Soc. 1993;70:763-6.

48. He H-P, Corke H. Oil and Squalene in Amaranthus grain and leaf. J Agric Food Chem. 2003;51:7913-20.

49. Valachovic M, Garaiova M, Holic R, Hapala I. Squalene is lipotoxic to yeast cells defective in lipid droplet biogenesis. Biochem Biophys Res Commun. 2016:469:1123-8.

50. El-Hagrassi AM, Ali MM, Osman AF, Shaaban M. Phytochemical investigation and biological studies of Bombax malabaricum flowers. Nat Prod Res. 2011; 25:141-51.

51. Wu C-R, Hseu Y-C, Lien J-C, Lin L-W, Lin Y-T, Ching H. Triterpenoid contents and anti-inflammatory properties of the methanol extracts of Ligustrum species leaves. Molecules. 2010;16:1-15.

52. Okoye NN, Ajaghaku DL, Okeke HN, llodigwe EE, Nworu CS, FBC O. BetaAmyrin and alpha-amyrin acetate isolated from the stem bark of Alstonia Boonei display profound anti-inflammatory activity. Pharm Biol. 2014;52: 1478-86.

53. Romero-Estrada A, Maldonado-Magaña A, González-Christen J, Bahena SM, Garduño-Ramírez ML, Rodríguez-López V, et al. Anti-inflammatory and antioxidative effects of six pentacyclic triterpenes isolated from the Mexican copal resin of Bursera copallifera. BMC Complement Altern Med. 2016;16:422.

54. Liu J, Xiong H, Cheng Y, Cui C, Zhang X, Xu L, et al. Effects of taraxasterol on ovalbumin-induced allergic asthma in mice. J Ethnopharmacol. 2013;148:787-93.
55. Wang S, Wang Y, Liu X, Guan L, Yu L, Zhang X. Anti-inflammatory and antiarthritic effects of taraxasterol on adjuvant-induced arthritis in rats. J Ethnopharmacol. 2016;187:42-8.

56. Zhang $\mathrm{X}$, Xiong $\mathrm{H}$, Liu L. Effects of taraxasterol on inflammatory responses in lipopolysaccharide-induced RAW 264.7 macrophages. J Ethnopharmacol. 2012;141:206-11.

57. Takasaki M, Konoshima T, Tokuda K, Masuda K, Arai Y, Shiojima K, et al. Anticarcinogenic activity of Taraxacum plant. II. Biol Pharm Bull. 1999;22:606-10.

58. Sharma K, Zafar R. Optimization of methyl jasmonate and $\beta$-cyclodextrin for enhanced production of taraxerol and taraxasterol in (Taraxacum officinale Weber) cultures. Plant Physiol Biochem. 2016;103:24-30.

\section{Submit your next manuscript to BioMed Central and we will help you at every step:}

- We accept pre-submission inquiries

- Our selector tool helps you to find the most relevant journal

- We provide round the clock customer support

- Convenient online submission

- Thorough peer review

- Inclusion in PubMed and all major indexing services

- Maximum visibility for your research

Submit your manuscript at www.biomedcentral.com/submit
Biomed Central 